\title{
Data-driven user behavioral modeling: from real-world behavior to knowledge, algorithms, and systems
}

\section{Special issue introduction}

\author{
Ludovico Boratto $^{1}$ - Eloisa Vargiu ${ }^{1}$
}

Published online: 20 January 2020

(C) Springer Science+Business Media, LLC, part of Springer Nature 2020

\section{Introduction}

Nowadays, we are inundated with data gathered from the users, both in the real- and in the digital-world. Data streams come from sensors (e.g., environmental) and devices (e.g., activity trackers, medical devices, and cameras) connected together according to Internet of Things (IoT) protocols, social media continuously updated with text, photos, and videos, as well as geolocalizated systems that send the user's location in real time. Thus, it is more and more required to mine those data searching for patterns and rules to guide intelligent systems to provide recommendations, feedback, suggestions, and nudge in a personalized and adaptive way. The goal is to create a snapshot, or profile, of the user by understanding her/his behavior when searching for a product, activities when near to a store, and the influence of a recommendation in a social media.

Data tells us the much of the user's story and we have to complement it through tools and techniques able to look for patterns that have to be turned into knowledge to guide intelligent algorithms and systems in making smarter recommendations. In other words, our goal is to transform data into knowledge to support and enhance the user's experience. Being interested in making recommender systems smarter and more responsive to user's needs, we first need to better understand the users. In that direction, one important requirement is to allow the users to provide feedback regarding the recommendations provided by the system. Moreover, we have to take into consideration the role of social media in influencing users' decision-making.

The focus of this special issue is to explore how data-driven behavioral modeling can be used to generate knowledge that can feed algorithms and systems.

Ludovico Boratto

ludovico.boratto@acm.org

Eloisa Vargiu

eloisa.vargiu@eurecat.org

1 Eurecat, Centre Tecnològic de Catalunya, Barcelona, Spain 


\section{In this special issue}

The nine articles published in this special issue propose innovative techniques that use different data sources in order to model user's behavior and extract knowledge from it. The research contributions advance the state of the art, considering different aspects and scenarios, ranging from content recommendation to community detection, by employing data coming from social media and mobile platforms.

Chouchani and Abed compare approaches for clustering Social Network actors into communities of interests, in their paper "Online Social Network Analysis: Detection of communities of interest" (Chouchani and Abed 2018). Specifically, in this survey the authors review and classify, in a novel way, the existing approaches and highlight the differences between them based on certain criteria.

The paper "Data-driven decision making in Critique-based Recommenders: from a critique to social media data", by Contreras and Salamó (2018), tackles the issue of the sources of data that are used to generate the recommendations and of how they can be used to provide users with effective recommendations. Specifically, the authors propose an algorithm, named HOR, that integrates several data sources, such as current user preferences (i.e., a critique), product descriptions, previous critiquing sessions by other users, and users' opinions expressed as ratings on social media web sites. The algorithm is then integrated with compatibility and weighting scores to turn user behavior into knowledge, and with a state-of-the-art approach, named HGR, to help both algorithms make smarter recommendations.

In their article "Exploiting recommendation confidence in decision-aware recommender systems" (Mesas and Bellogín 2018), Mesas et al. analyze the problem of when recommendations should be produced, according to the confidence that an algorithm has of generating effective results. The authors propose a taxonomy of techniques that can be applied to understand if a recommendation should be generated and a study of different evaluation metrics, mainly to compare the precision of the system and its coverage.

Rook et al., in their article "Engagement in Proactive Recommendations: The Role of Recommendation Accuracy, Information Privacy Concerns, and Personality Traits" (Rook et al. 2018), explore to what extent user's engagement in proactive recommendation scenarios is influenced by the accuracy of recommendations, concerns with information privacy, and trait personality. Thanks to this study, the authors highlight that the concerns about information privacy positively influence user's engagement with proactive recommendations. Moreover, they show that recommendation accuracy influences the engagement of users with privacy concerns.

The paper, "Predicting future personal life events on Twitter via recurrent neural networks", by Khodabakhsh et al. (2018), predicts a future personal life event that a user will be posting about on Twitter, solely based on the past tweets. The work employs recurrent neural networks (classification and generation architectures), in order to determine the future personal life event of a user.

Ali et al. propose a topic- and sentiment-aware microblog summarization for Twitter, in their article "Topic and Sentiment Aware Microblog Summarization for Twitter" (Ali et al. 2018). Specifically, the authors pre-process and semantically enrich microblog posts, to then extract the topics (through LDA) and sentiments expressed by each post. A sentimentbased Word Graph for each topic is built and the graph is clustered to extract different topical aspects. Finally, different methods are applied to summarize each topical aspect individually, and aggregate all aspect-level summaries. 
In their article "From mobility patterns to behavioural change: Leveraging travel behaviour and personality profiles to nudge for sustainable transportation", Anagnostopoulou et al. (2018) present an approach to persuade users to change their mobility behavior and make more sustainable choices. The approach uncovers users' mobility patterns and use of transportation modes through pervasive mobile sensing. After profiling the users, personalized interventions that nudge users to adopt sustainable transportation habits are produced. These interventions are embedded in a route planning application for smartphones.

The paper "A knowledge-based multi-criteria collaborative filtering approach for discovering services in mobile cloud computing platforms", by Colombo-Mendoza et al. (2018), proposes a knowledge-based collaborative filtering recommender system aimed at discovering services in the MobiCloUP! mobile cloud computing platform for services-based development. Specifically, the approach employs a knowledge-based technique that exploits Semantic Web rule-based reasoning. The preferences of developers are interpreted as sets of constraints, and a Multi-Criteria Decision Making (MCDM) method is employed to solve the multi-criteria recommendation problem.

The last paper of this special issue, titled "Privacy-Preserving Shared Collaborative Web Services QoS Prediction”, by Liu et al. (2018), propose a novel approach to Collaborative Web services QoS prediction (CQoSP), named "shared CQoSP". The approach allows multiple parties to share their data, in order to produce more effective results with respect to the case in which a single party is allowed to. Data sharing is made possible thanks to a privacypreserving framework based on differential privacy, which enables the prediction without any data leaking.

\section{Conclusions}

Modeling user behavior with data-driven approaches is a topic of central interest to gather actionable knowledge for algorithms and systems. The papers included in this special issue cover several topics and present some of the key directions in this vibrant and rapidly expanding area of research and development. We hope the set of selected papers provides the community with a better understanding of the current directions, and that they inspire readers with possible areas to focus on in their future research.

Acknowledgments We thank all the authors for considering this special issue as an outlet to publish their research results in the area of data-driven behavioral modeling. We also would like to thank the referees who provided very useful and thoughtful feedback to the authors. Finally, we express our gratitude to the Editors-in-Chief, Professor Kerschberg and Professor Ras, for their kind support, advice, and encouragements throughout the preparation of this special issue.

\section{References}

Ali, S.M., Noorian, Z., Bagheri, E., Ding, C., Al-Obeidat, F. (2018). Topic and sentiment aware microblog summarization for twitter. Journal of Intelligent Information Systems. https://doi.org/10.1007/s10844018-0521-8.

Anagnostopoulou, E., Urbančič, J., Bothos, E., Magoutas, B., Bradesko, L., Schrammel, J., Mentzas, G. (2018). From mobility patterns to behavioural change: leveraging travel behaviour and personality pro- 
files to nudge for sustainable transportation. Journal of Intelligent Information Systems. https://doi.org/ 10.1007/s10844-018-0528-1.

Chouchani, N., \& Abed, M. (2018). Online social network analysis: detection of communities of interest. Journal of Intelligent Information Systems. https://doi.org/10.1007/s10844-018-0522-7.

Colombo-Mendoza, L.O., Valencia-García, R., Colomo-Palacios, R., Alor-Hernández, G. (2018). A knowledge-based multi-criteria collaborative filtering approach for discovering services in mobile cloud computing platforms. Journal of Intelligent Information Systems. https://doi.org/10.1007/s10844-0180527-2.

Contreras, D., \& Salamó, M. (2018). Data-driven decision making in critique-based recommenders: from a critique to social media data Journal of Intelligent Information Systems. https://doi.org/10.1007/s10844018-0520-9.

Khodabakhsh, M., Kahani, M., Bagheri, E. (2018). Predicting future personal life events on twitter via recurrent neural networks. Journal of Intelligent Information Systems.

Liu, A., Shen, X., Xie, H., Li, Z., Liu, G., Xu, J., Zhao, L., Wang, F.L. (2018). Privacy-preserving shared collaborative web services qos prediction. Journal of Intelligent Information Systems. https://doi.org/10. 1007/s10844-018-0525-4.

Mesas, R.M., \& Bellogín, A. (2018). Exploiting recommendation confidence in decision-aware recommender systems. Journal of Intelligent Information Systems. https://doi.org/10.1007/s10844-018-0526-3.

Rook, L., Sabic, A., Zanker, M. (2018). Engagement in proactive recommendations: the role of recommendation accuracy, information privacy concerns, and personality traits. Journal of Intelligent Information Systems. https://doi.org/10.1007/s10844-018-0529-0.

Publisher's note Springer Nature remains neutral with regard to jurisdictional claims in published maps and institutional affiliations. 